\title{
Promoção e avaliação da atitude de Vigilância Nutricional na Atenção Básica à Saúde de municípios das bacias Piracicaba-Capivari
}

\author{
Andreza Alves Camargo ${ }^{1}$, Maria Rita Marques de Oliveira ${ }^{2}$, Roselene Valota Renosto ${ }^{3}$, \\ Carla Maria Vieira ${ }^{4}$
}

Considerando a importância das ações de alimentação e nutrição na Atenção Básica à Saúde para a Segurança Alimentar e Nutricional da população, o objetivo foi apresentar e discutir uma experiência educativa de promoção da atitude de vigilância nutricional, desenvolvida em oficinas com trabalhadores da atenção básica de onze municípios do interior paulista. Trata-se de um relato de experiência que constou da realização e observação de oficinas com registro em diário de campo, seguidas de processo avaliativo, a partir de questionários semi-estruturados, com profissionais das equipes de atenção básica dos municípios. Os resultados foram organizados e analisados sob a forma de quatro categorias. A metodologia participativa e vivencial das oficinas foi considerada adequada, porém demandou acolhimento dos sentimentos revelados pelos participantes, tais como constrangimento e inibição, superados após o desenvolvimento de dinâmicas de grupo. Dentre os temas trabalhados a antropometria teve destaque, indicando a falta de padronização e conhecimento técnico. A atitude de vigilância nutricional foi reconhecida pelos profissionais de saúde como importante para lhes dar respaldo nas práticas específicas de nutrição, contribuindo para a segurança alimentar e nutricional da população.

Palavras-chave: políticas públicas de saúde, vigilância nutricional, capacitação de recursos humanos.

\section{Promotion and evaluation of the Nutritional Surveillance in Primary Care of the Piracicaba-Capivari watersheds}

Considering the importance of food and nutritional surveillance as part of a community's basic health care, our objective was to expose and discuss the educational experience of eleven municipalities of the state of São Paulo geared to build an attitude of nutritional security. The accounts were made in the form of workshops with the contribution of basic health care workers of each municipality, based on daily field worksheets and followed by an evaluation process, which used a semi-structured questionnaire. The results were classified into four categories. The methodology, based on the actual participation in the workshops was considered adequate, although some exposure and removal of personal inhibitions were necessary. These barriers were left behind with the aid of group dynamic exercises. Among the themes treated, anthropometry was highlighted because of insufficient technical know-how and standardization. An attitude towards nutritional surveillance was recognized by the health-care professionals as an important basis for the nutritional practice in order to attain food and nutritional security.

Key-words: public heath policies, nutritional surveillance, resource human qualification.

\footnotetext{
${ }^{1}$ Nutricionista formada pelo curso de Nutrição da Faculdade de Ciências da Saúde, Universidade Metodista de Piracicaba (UNIMEP), Piracicaba, SP.

${ }^{2}$ Profa. Dra. do Instituto de Biociências, Universidade Estadual Paulista “Júlio de Mesquita Filho” (UNESP), Botucatu, SP.

${ }^{3}$ Docente do curso de Nutrição, Faculdade de Ciências da Saúde, Universidade Metodista de Piracicaba (UNIMEP), Piracicaba, SP.

${ }^{4}$ Docente do curso de Nutrição, Faculdade de Ciências da Saúde, Universidade Metodista de Piracicaba (UNIMEP), Piracicaba, SP. Correspondência: Rodovia do Açúcar, km 156, CEP 13400-901, Piracicaba, SP. Tel: (19) 3124 1583. E-mail: cmvieira@unimep.br
} 


\section{INTRODUÇÃO}

O Brasil como outros países em desenvolvimento, tem apresentado profundas modificações no perfil nutricional de sua população, fruto de um processo conhecido como transição nutricional, ou seja, redução da prevalência de desnutrição e aumento da obesidade [1]. Os problemas decorrentes de uma alimentação inadequada expõem a população a graves danos à saúde, principalmente entre crianças e adolescentes em algumas regiões e em grupos menos privilegiados [2].

Nesse cenário de mudanças no padrão de problemas nutricionais, o estado nutricional emerge como um forte indicador capaz de refletir basicamente o consumo alimentar e o estado de saúde dos indivíduos [2]. Assim, torna-se fundamental a adoção de ações de monitoramento no sentido de garantir a segurança alimentar e nutricional, de maneira a identificar determinantes de tais agravos, conforme a dinâmica do perfil nutricional dos indivíduos [3].

Organismos internacionais salientam a necessidade de estabelecer medidas de promoção da nutrição para o desenvolvimento das nações. No entanto, há reconhecimento das dificuldades dos governos no controle dos problemas ligados à nutrição. Agências, como a Organização Mundial de Saúde (OMS), a Organização Pan-Americana da Saúde (OPAS) e o Fundo das Nações Unidas para a Infância (UNICEF) têm dado grande ênfase ao incentivo à implantação de sistemas de vigilância alimentar e nutricional [4].

No Brasil, o Sistema Nacional de Vigilância Alimentar e Nutricional (SISVAN) é previsto na Norma Operacional Básica do Sistema Único de Saúde de 1996 (NOB-SUS 96) e foi concebido sobre três eixos principais que se propõem a formular políticas públicas, planejar, acompanhar e avaliar programas sociais relacionados à alimentação e nutrição bem como avaliar a eficácia das ações governamentais.

Na saúde, o SISVAN é um instrumento para obtenção de dados de monitoramento do estado nutricional e do consumo alimentar das pessoas que frequentam as Unidades Básicas de Saúde (UBS), possibilitando que o Sistema Único de Saúde promova estratégias para prevenir e tratar os desvios nutricionais sejam eles o baixo peso ou a obesidade e, assim, orientar as políticas públicas em todas as esferas de governo [5].
Neste sentindo, o SISVAN tem por objetivo atualizar o diagnóstico dos problemas mais relevantes relacionados à alimentação e nutrição no país, identificar as regiões e os grupos populacionais sob risco nutricional, reunir dados que possibilitem identificar e ponderar as causas desses problemas e oferecer subsídios ao planejamento e à execução de medidas para a melhoria da situação alimentar [5].

Os dados gerados por esse sistema têm, portanto, o papel de auxiliar os gestores públicos na gestão de políticas de alimentação e nutrição. No entanto, o recolhimento dessas informações pelos diversos programas e setores (agricultura, economia, planejamento, educação, saúde...), que deveriam subsidiar tais políticas governamentais [5,6], ainda não se efetivou de forma satisfatória no país.

O monitoramento da situação alimentar e nutricional da população brasileira por meio do SISVAN também está contemplado na terceira diretriz da Política Nacional de Alimentação e Nutrição (PNAN) [6,7]. Aprovada em 1999 pelo Conselho Nacional de Saúde, a PNAN tem como propósito a garantia da qualidade dos alimentos colocados para o consumo no país, a promoção de práticas alimentares saudáveis e a prevenção e o controle de distúrbios nutricionais [6]. Sua implantação é responsabilidade das três esferas de governo (nacional, estadual e municipal) e dos diversos atores e setores sociais, cabendo ao Ministério da Saúde a sua coordenação nacional [6,7].

As diretrizes três e sete da PNAN, preconizam ainda o desenvolvimento e a capacitação de recursos humanos em Vigilância Alimentar e Nutricional para os profissionais de saúde envolvidos com o SISVAN. As atividades educativas neste campo devem envolver os estados, regionais de saúde, municípios, e outros parceiros importantes no processo, como por exemplo, projetos de pesquisa e extensão universitária [ ${ }^{6}$.

Diversos estudos [8,9] sobre capacitação de profissionais da saúde mostram que os envolvidos nesse processo trazem consigo conceitos e informações leigas e muitas vezes não verdadeiras. Em geral, médicos e enfermeiros têm formação deficiente em nutrição, visto que no Brasil, são poucas as escolas de medicina e enfermagem que dão atenção ao ensino dessa área. Além disso, não há nutricionista em muitos serviços de atendimento primário e a alta prevalência de doenças crônicas exige que profissionais de saúde estejam preparados para orientar os pacientes em relação aos fatores de proteção às doenças crônicas, entre os quais, está a alimentação. 
Boog [9] em estudo sobre implementação da educação nutricional em serviços públicos de saúde revelou que médicos e enfermeiros apresentam dificuldades para identificar e lidar com problemas alimentares. Frequentemente tendem a considerar estes como uma questão do cotidiano ou atribuir a causa deles apenas a fatores econômicos. As enfermeiras, mais do que os médicos, consideram que o ensino de nutrição foi insatisfatório na graduação e julgam insatisfatórios os conhecimentos que possuem nessa área.

Experiências locais de educação em saúde envolvendo habilidades no campo da vigilância nutricional têm sido reconhecidas. Atividades de capacitação para profissionais da atenção básica em alimentação e nutrição desenvolvidas em várias regiões brasileiras são exemplos nesse sentido. A necessidade de melhorar a oferta dos serviços de vigilância nutricional na atenção básica justifica os projetos de formação, tendo em vista a necessidade de atualização dos profissionais de saúde ${ }^{[10]}$.

A promoção da atitude de vigilância por parte da equipe da atenção básica é tratada em publicações da Coordenção Geral da Política de Alimentação e Nutrição (CGPAN) como uma questão relevante na implementação do SISVAN. Atitude de vigilância no sentido de compromisso e postura profissional tendo em vista o cuidado com o estado nutricional da população assistida pelo SUS. temos que:

Segundo a descrição do Manual do SISVAN

Atitude de vigilância é ter um olhar diferenciado para cada indivíduo, para cada grupo, para cada fase do ciclo de vida. É usar a informação gerada rotineiramente não só para retroalimentar o Sistema de Vigilância Alimentar e Nutricional e subsidiar as programações locais e as instâncias superiores, mas também usá-la de forma imediata para repensar a prática e qualificar a assistência prestada àqueles indivíduos que diariamente são atendidos na rede de saúde (2004, p. 11)[].

Essas atividades exigem, no entanto, constante avaliação para identificar os resultados desse processo. Diante dessas considerações e justificativas, o presente estudo teve como objetivo apresentar e discutir uma experiência educativa de promoção da atitude de vigilância nutricional, desenvolvida com trabalhadores da atenção básica de onze municípios do interior paulista.

\section{METODOLOGIA}

O projeto "Avaliação da implementação das ações de Vigilância Alimentar e Nutricional no âmbito da Atenção Básica do SUS na região das Bacias Piracicaba-Capivari", o qual deu origem a este artigo, foi concebido no início do ano de 2007. Inicialmente participaram gestores, profissionais da atenção básica e docentes universitários, todos com acúmulo de experiências e envolvimento em projetos locais de segurança alimentar e vigilância nutricional, de um município de grande porte no interior paulista.

O projeto de pesquisa foi aprovado pelo Comitê de Ética em Pesquisa da Universidade Metodista de Piracicaba sob o protocolo no 83/05, sendo que todos os sujeitos foram previamente informados sobre os propósitos da pesquisa e assinaram um termo de consentimento concordando em participar do trabalho.

Em virtude da constituição da Comissão Regional de Segurança Alimentar e Nutricional Sustentável (CRSANS), vinculada ao Conselho Estadual de Segurança Alimentar e Nutricional, que ocorria no mesmo período, a coordenação do projeto acima referido propôs a extensão do mesmo aos municípios participantes da CRSANS, inseridos no território geográfico que compõe as bacias dos rios Piracicaba e Capivari ${ }^{5}$. O grupo de trabalho foi, portanto, ampliado com reuniões para planejamento da pesquisa e da intervenção que ocorreram na etapa inicial.

A "pactuação" do projeto com os gestores municipais constituiu a etapa seguinte, realizada por meio de visitas de representantes do grupo aos Secretários Municipais de Saúde. Posteriormente, agrupados em municípios de mais fácil acesso, coordenadores de unidades de saúde, selecionados pelos gestores e participantes do projeto planejaram as atividades de intervenção e posterior avaliação.

\footnotetext{
5 Trata-se de região agroindustrial, cuja cultura predominante é a cana de açúcar, seguida das pastagens, sendo bem tímida a produção de grãos, frutas e de hortaliças. Em 2008, a expectativa de vida média da região era de 72,95 anos e a taxa de alfabetização de 94,95 [11]. O Índice de Desenvolvimento Humano Municipal (IDH-M) dos municípios participantes superava 0,800 , sendo compatível com os índices dos países mais desenvolvidos. A distância desses municípios dos grandes centros urbanos do Estado de São Paulo está entre 100 e 200 $\mathrm{km}$, com malha viária que garante bom acesso às diferentes regiões paulistas.
} 
Foram convidados a participar do trabalho 13 municípios, agrupados conforme o número de habitantes: Grupo A, entre 100.000 e 500.000 habitantes (dois municípios: GA-1, GA-2), Grupo B, entre 10.000 e 50.000 habitantes (seis municípios: GB1, GB-2, GB-3, GB-4, GB-5, GB-6), Grupo C, entre 5.000 e 10.000 habitantes (um município: GC-1) e Grupo D, com menos de 5.000 habitantes (dois municípios: GD-1, GD-2). Dos 13 municípios, 11 participaram das oficinas somando um total de 35 equipes de saúde.

$\mathrm{Na}$ rede básica de saúde, a Estratégia Saúde da Família estava parcialmente implantada na região. Nos municípios estudados foram encontradas Unidades Básicas de Saúde e Unidades Mistas de Saúde (UMS), além de algumas Unidades de Saúde da Familia (USF).

O material aqui apresentado complementa outros trabalhos que versam sobre os resultados da pesquisa e intervenção do projeto original, em fase de publicação. Este artigo contempla a análise de um conjunto de dados de caráter qualitativo, obtidos em duas etapas distintas: fase I - acompanhamento das atividades de planejamento e execução das atividades educativas; fase II - avaliação, seis meses após a realização das atividades educativas.

\section{Fase I - As oficinas}

O conjunto de oficinas ocorreu no segundo semestre de 2007. Foram formados três grupos de oficinas (G1, G2 e G3). Cada grupo foi formado por profissionais das unidades de atenção básica de municípios territorialmente próximos, com variação no número de encontros (quatro a oito encontros), em função da disponibilidade das equipes.

O objetivo principal das oficinas estava voltado à promoção da prática de vigilância nutricional, prevenção e controle dos desvios nutricionais no contexto do Sistema Único de Saúde, objetivo este identificado com as diretrizes da Política Nacional de Alimentação e Nutrição do Ministério da Saúde.

Para a execução do trabalho foi priorizado o processo participativo. O método de trabalho adotado seguiu a proposta do Ministério da Saúde para a Educação Permanente, pautada na educação problematizadora, dialógica e participante $[12,13,14]$. O Manual de Vigilância Alimentar e Nutricional do SISVAN [7] e o Guia Alimentar da População Brasileira [15] serviram como apoio para organização dos conteúdos trabalhados.
O modelo de oficinas da proposta de trabalho de Afonso [12], oriundo do campo da psicologia social, foi adotado como referencial teórico e metodológico nesta fase do trabalho. Esta proposta de oficina é desenvolvida com base no modelo de grupo operativo e de dinâmicas de grupo.

Segundo a autora ${ }^{[12]}$, oficina é

...um trabalho estruturado em grupos, independente do número de encontros, sendo focalizado em torno de uma questão central que o grupo se propõe a elaborar, dentro ou fora de um contexto institucional. A elaboração que se busca na oficina não se restringe a uma reflexão racional, mas envolve os sujeitos de maneira integral, seus sentimentos, formas de pensar e de agir (2006, p. 67).

Esse modelo tem como objetivo conhecer as ideias, crenças e sentimentos dos participantes, tornando possível a comunicação, o desenvolvimento da autonomia e o estímulo à criatividade dos participantes. A aprendizagem coletiva com os educandos e educadores em conjunto é proposta para que ambos possam enfrentar e se relacionar de forma dinâmica com dada problemática, que por sua vez é influenciada por fatores sociais, culturais e psíquicos. Nessa abordagem incentivam-se mudanças de pensamento e estimulam-se ações que se apliquem na prática cotidiana ${ }^{[12] .}$

As oficinas foram coordenadas por uma nutricionista e duas acadêmicas de nutrição, envolvidas desde o projeto original. Para a preparação das oficinas ocorreram reuniões semanais das educadoras com uma docente universitária, nutricionista e educadora em saúde, igualmente integrante do grupo do projeto. A cada encontro semanal um roteiro de oficina era elaborado a partir das questões discutidas sobre a dinâmica grupal, os conteúdos e estratégias metodológicas de condução e avaliação.

Durante o desenvolvimento das oficinas ocorreram os registros em diário de campo com o consentimento dos participantes. Foram registradas questões relativas à execução das oficinas, percepções e diálogos estabelecidos no processo, para posterior análise.

Cada grupo estabeleceu seu próprio ritmo de trabalho com expectativas e interesses particulares que compuseram demandas de aprendizagem distintas. 
Desta forma, foram trabalhados no mínimo quatro e no máximo oito temas nas oficinas.

Os temas trabalhados e o número de participantes estão ilustrados no Quadro. Um encontro com um dos grupos foi ocupado com uma oficina específica de avaliação final. E outro grupo também foi dedicado a um encontro para elaboração de "contrato grupal", tendo em vista dificuldades de compreensão da proposta, assim como a dispersão observada durante os encontros iniciais.

Dentre os representantes das equipes de Unidades Básicas de Saúde que participaram das oficinas os agentes comunitários de saúde prevaleceram. Além deste profissional, enfermeiras, auxiliares e técnicos de enfermagem também participaram do processo educativo.

\section{Fase II - A avaliação}

De um total de onze unidades de saúde que enviaram profissionais para participar das oficinas apenas cinco participaram das entrevistas de avaliação. As equipes descartadas na segunda fase do trabalho haviam sofrido algum tipo de mudança de local e principalmente de membros das equipes ou gestores. A fase de avaliação ocorreu após o período eleitoral nos municípios alterando significativamente as equipes e a política de saúde local, o que refletiu no projeto original como um todo, no primeiro semestre de 2008 .

\begin{tabular}{|c|c|c|c|c|}
\hline \multirow{2}{*}{ Tema } & \multicolumn{4}{|c|}{ Número de participantes nas oficinas } \\
\hline & Grupo 1 & Grupo 2 & Grupo 3 & TOTAL \\
\hline 1 - Alimentação Saudável e Vigilância Nutricional do Adulto & 12 & 11 & 0 & 23 \\
\hline 2 - Vigilância Nutricional da Criança & 6 & 10 & 0 & 16 \\
\hline 3 - Alimentação e Desenvolvimento da Criança & 5 & 12 & 0 & 17 \\
\hline 4 - Vigilância Alimentar e Nutricional da Gestante & 10 & 4 & 40 & 54 \\
\hline 5 - Alimentos enriquecidos & 6 & 8 & 0 & 14 \\
\hline 6 - Vigilância nutricional do Idoso & 4 & 0 & 0 & 4 \\
\hline 7 - Contrato Grupal & 0 & 0 & 42 & 42 \\
\hline 8 - Criança de 0 a 2 anos de idade em fase de amamentação & 0 & 0 & 34 & 34 \\
\hline 9 - Avaliação & 0 & 10 & 0 & 10 \\
\hline Total de participações nas oficinas & 43 & 55 & 116 & 214 \\
\hline
\end{tabular}

Quadro. Temas das oficinas e numero de participantes distribuídos por grupos de municípios da região das Bacias Piracicaba-Capivari, 2007.

Foram realizadas visitas às unidades de saúde que concordaram em participar da avaliação após a primeira fase do projeto. Nem sempre o profissional que foi entrevistado havia participado das oficinas, no entanto, foi garantido que o mesmo havia tido pelo menos contato com algum colega que foi integrante do grupo das oficinas de vigilância alimentar e nutricional.
Para efeito de análise, os sujeitos participantes desta etapa do trabalho foram identificados com uma letra referente à sua profissão seguida por códigos indicativos do município ${ }^{6}$. Foram entrevistados 15 sujeitos distribuídos em cinco unidades de saúde de cinco municípios participantes do projeto original (Tabela).

${ }^{6} \mathrm{E}=$ enfermeira; $\mathrm{A}=$ auxiliar de enfermagem; $\mathrm{T}=$ técnico de enfermagem; $\mathrm{C}=$ agente comunitário de saúde; $\mathrm{M}=$ médico. 
Tabela. Caracterização dos sujeitos participantes das entrevistas de acordo com sexo, idade e profissão, na Região das Bacias Piracicaba-Capivari, 2008.

\begin{tabular}{|c|c|c|c|c|}
\hline \multirow{3}{*}{ Características } & \multicolumn{2}{|c|}{ Sexo } & \multirow{2}{*}{\multicolumn{2}{|c|}{ Total $(n=15)$}} \\
\hline & \multirow{2}{*}{$\begin{array}{c}\text { Feminino }(n=12) \\
n\end{array}$} & \multirow{2}{*}{$\begin{array}{c}\text { Masculino }(n=3) \\
n\end{array}$} & & \\
\hline & & & $n$ & $\%$ \\
\hline \multicolumn{5}{|l|}{ Faixa Etária } \\
\hline 21-30 anos & 4 & 1 & 5 & 33 \\
\hline $31-40$ anos & 4 & 1 & 5 & 33 \\
\hline $41-50$ anos & 4 & 1 & 5 & 33 \\
\hline Total & 12 & 3 & 15 & 100 \\
\hline \multicolumn{5}{|l|}{ Profissão } \\
\hline Enfermeira & 3 & 0 & 3 & 20 \\
\hline Auxiliar de Enfermagem & 3 & 2 & 5 & 33 \\
\hline Técnico em Enfermagem & 3 & 0 & 3 & 20 \\
\hline Agente comunitário de saúde & 3 & 0 & 3 & 20 \\
\hline Médico & 0 & 1 & 1 & 7 \\
\hline Total & 12 & 3 & 15 & 100 \\
\hline \multicolumn{5}{|l|}{ Procedência } \\
\hline GB1 & 4 & 1 & 5 & 33 \\
\hline GB6 & 2 & 0 & 2 & 13 \\
\hline GB2 & 2 & 1 & 3 & 20 \\
\hline GD2 & 2 & 1 & 3 & 20 \\
\hline GB5 & 2 & 0 & 2 & 13 \\
\hline Total & 12 & 3 & 15 & 100 \\
\hline
\end{tabular}

Nessa fase foram identificadas opiniões dos profissionais por meio de questionários semiestruturados com questões abertas aplicados pelas acadêmicas que participaram previamente das oficinas. Para este artigo, o conjunto de dados registrados nas duas etapas foram sistematizados em forma de relato de experiência.

Quatro categorias foram organizadas para a apresentação e discussão dos resultados: Os temas das oficinas de Vigilância Alimentar e Nutricional; A antropometria e a utilização do Guia alimentar e do Manual do SISVAN no apoio à atitude de vigilância; A metodologia das oficinas $e$ as dificuldades operacionais; O campo de conhecimentos da nutrição e a inserção do nutricionista na atenção básica.

\section{RESULTADOS E DISCUSSÃO}

1. Os conteúdos das oficinas e a promoção da atitude de Vigilância Alimentar e Nutricional na atenção básica em saúde
Durante as oficinas foi identificado que os profissionais de saúde tinham interesse em aprofundar seus conhecimentos no campo da nutrição e alimentação. Interesse este vinculado à prática cotidiana nas unidades de atenção primária, particularmente em relação ao trabalho de orientação de mães visando o melhor desenvolvimento $\mathrm{e}$ crescimento infantil.

$\mathrm{Na}$ oficina cujo tema foi vigilância nutricional e alimentar da criança o grupo de profissionais demonstrou interesse sobre a importância do monitoramento nutricional das crianças menores de sete anos. Carência de nutrientes e biodisponibilidade também foram aspectos que marcaram o envolvimento dos profissionais nas atividades educativas voltadas para este tema7.

\footnotetext{
7 Particularmente, a ação do cálcio como inibidor da absorção do ferro, fator que contribui para a susceptibilidade da criança a desenvolver anemia ou dificultar seu tratamento despertou o debate e interesse dos grupos.
} 
$\mathrm{Na}$ fase de avaliação desta experiência os conteúdos de nutrição infantil foram relembrados nas entrevistas. Esse resultado demonstra certa aproximação da teoria com a prática no desenvolvimento da experiência.

"Gostei de saber sobre a absorção do cálcio, pois sabia que não era bom dar mamadeira para a criança após refeição e descobri o porquê...” (AGB1).

Os conteúdos de nutrição e alimentação do idoso ocuparam igualmente o interesse dos participantes das oficinas. Os profissionais demonstraram alcançar o aprofundamento no tema sendo capazes de reconhecer que algumas alterações fisiológicas deste ciclo de vida interferem na maneira de se alimentar. E, por conseguinte, a importância de conhecer as necessidades nutricionais e os parâmetros de avaliação do estado nutricional do idoso.

O conjunto de manifestações sobre esses e outros conteúdos trabalhados nas oficinas revelou que as vivências de aprendizagem coletiva promoveram a oportunidade aos participantes de alcançar um novo patamar de domínio teórico prático. Sinais de empoderamento para a solução das questões relacionadas à alimentação e nutrição, à atitude de vigilância e segurança alimentar foram observados ao longo da experiência.

Empoderamento é entendido como o processo de capacitação para a aquisição de poder técnico e político por parte dos indivíduos e da comunidade. É necessário que as pessoas estejam abertas às mudanças de seus hábitos antigos para que o processo de empoderamento seja eficaz, o que requer um comprometimento permanente e contínuo de pensamentos críticos, os quais sejam seguidos de ações [16,17].

Ao mesmo tempo em que eram percebidos sinais de apropriação de novos conhecimentos, os participantes reconheciam também que isso não solucionaria a dificuldade encontrada em seu cotidiano, em relação às mudanças de atitudes e práticas alimentares dos pacientes. $\mathrm{Na}$ visão de alguns profissionais que participavam das oficinas o significado de alimentação saudável está vinculado a um privilégio de uma determinada classe social e, portanto, fora do alcance da maioria da população que é orientada na rede pública de atenção básica.

Em artigo de revisão, significados das dietas e das mudanças de comportamento alimentar no aconselhamento nutricional, foram identificados, de maneira semelhante ao encontrado nesta experiência.
Pacientes que não conseguem seguir as orientações nutricionais apelam para a justificativa de que a alimentação saudável tem elevado valor monetário e demanda maior tempo de preparo ${ }^{[18]}$.

A dificuldade de orientação dos pacientes para a promoção das mudanças nas práticas alimentares foi uma questão também ressaltada na avaliação do processo educativo. Em entrevistas da segunda fase, no contexto das unidades de saúde, depoimentos revelaram a dificuldade da aproximação da teoria e da prática.

"... na prática o que aprendeu na teoria é muito difícil. Nem sempre podemos trabalhar com o que foi passado..." (AGB1).

Durante o processo educativo o grupo de coordenadores das oficinas levou em consideração a necessidade de aproximar, o máximo possível, as questões teóricas e práticas. Por meio do desenvolvimento da prática culinária o grupo identificou o potencial desta estratégia no processo de ensino aprendizagem como forma de manifestação dos aspectos subjetivos e socioculturais da alimentação presentes no grupo. A elaboração coletiva da prática culinária seguida de uma reflexão promoveu ao grupo a identificação de novas possibilidades para apoiar a difícil tarefa de orientação nutricional no cotidiano da atenção básica à saúde.

Para o desenvolvimento do tema "alimentos enriquecidos" a prática culinária foi escolhida como estratégia mediadora do processo de aprendizagem. Preparações de aproveitamento dos alimentos foram preparadas pelos participantes, com simplicidade, baixo custo e reprodutibilidade. $\mathrm{O}$ objetivo de encontrar possibilidades de incrementar a oferta de micronutrientes e fibras na alimentação de crianças, de adultos e idosos foi apresentado como forma de vincular a atividade ao tema proposto.

No decorrer das oficinas foram observados resultados significativos da prática culinária. Com o apoio de um receituário culinário organizado pelas coordenadoras das oficinas, os participantes manifestaram a possibilidade de ampliar a variação do cardápio cotidiano, assim como discutir aspectos ambientais vinculados ao consumo excessivo de alimentos industrializados, ao combate ao desperdício e à produção de lixo doméstico.

$\mathrm{Na}$ etapa de avaliação das oficinas a estratégia de prática culinária foi relembrada pelos entrevistados. Em seus discursos foi possível identificar o gosto pelas estratégias práticas e 
aplicabilidade no contexto da atenção básica em saúde.

"Gostei das partes práticas, chamaram mais a minha atenção" (TGB2). (AGB5).

"As receitas já foram passadas para os pacientes"

"Aprendi sobre desnutrição, obesidade, sobre alimentação saudável com a oficina dos alimentos enriquecidos..." (AGB6).

“... como orientar em relação à alimentação dos hipertensos, diabéticos e outras doenças...” (EGB6).

Um receituário culinário elaborado pelo grupo de educadoras teve como objetivo divulgar receitas de preparações ricas em ingredientes de elevado valor nutricional. Material semelhante foi recentemente lançado pela Secretaria de Desenvolvimento Social e Combate à Fome, preenchendo de certa maneira essa lacuna sentida no decorrer do processo educativo. O folder intitulado "Arroz e Feijão" [19] que divulga informações sobre os benefícios nutricionais do arroz com feijão, incentiva o consumo de produtos que valorizam a cultura nacional e promove a educação alimentar e nutricional.

Em estudo semelhante Silva et al. [20] identificaram que as capacitações têm como objetivo gerar mudanças na atuação do profissional, melhorando a resolutividade de suas ações. A participação do profissional é capaz de transformar a sua prática com o desenvolvimento de novos conhecimentos e habilidades que podem ser empregados e compartilhados com a equipe.

\section{A antropometria e a utilização do Guia alimentar e do Manual do SISVAN no apoio à atitude de vigilância}

A teoria e a prática de antropometria ocuparam um papel de destaque nessa experiência. No processo de desenvolvimento das oficinas de vigilância nutricional a abordagem de conteúdos sobre antropometria da criança, da gestante e do adulto causou entusiasmo em alguns encontros e polêmica em outros.

Os profissionais demonstraram o desejo de dominar técnicas de aferição das medidas antropométricas, utilização das curvas de crescimento da criança e interpretação dos dados de altura e peso, alegando pouco conhecimento sobre o assunto. Por outro lado, participantes envolveram-se em discussões que indicavam a falta de padronização na coleta de dados antropométricos nas unidades básicas de saúde.

A dinâmica realizada para tratar desse assunto, onde os próprios participantes eram sujeitos de um exercício de antropometria (peso, estatura, circunferência de cintura e cálculo do Índice de Massa Corporal - IMC) proporcionou o reconhecimento da padronização das técnicas de antropometria proposta no manual do SISVAN [7]. Deste modo, percebemos que os sujeitos correlacionaram essa atividade à importância da atitude de vigilância nutricional e compreenderam os parâmetros para o diagnóstico nutricional individual e coletivo do adulto.

Em um estudo feito por Fernandez [21] profissionais de saúde relataram sentir a necessidade de capacitação em função da falta de informações técnicas atualizadas e padronizadas no campo da nutrição. Atividade de treinamento foi relatada por este autor que defende a ideia de respeitar as atribuições específicas de cada profissional, na organização das atividades educativas.

A partir da análise do conjunto dos conteúdos dos depoimentos, na fase avaliativa desta experiência, foi reconhecido positivamente o tema antropometria. O reconhecimento do tema ocorreu a partir de uma experiência educativa que alcançou certo aprendizado, tanto em termos de habilidade prática, quanto um saber voltado para a satisfação pessoal, ilustrado pela fala dos entrevistados.

"O que me recordo foi da oficina de alimentação de crianças, a curva de crescimento que aprendi” (CGB1).

"Gostei muito de aprender sobre a antropometria, circunferências...” (EGD2).

A utilização do manual de antropometria e vigilância nutricional do SISVAN, durante as oficinas, fez emergir outro aspecto vinculado ao tema antropometria: as equipes de atenção básica em saúde envolvidas nesta experiência apresentavam desconhecimento em relação à utilização deste material, assim como do Guia Alimentar [15]. Os participantes demonstraram surpresa e entusiasmo ao entrar em contato com o conteúdo das publicações da CGPAN durante os encontros.

O Guia Alimentar para a População Brasileira [15] é um documento oficial do Ministério da Saúde, elaborado para subsidiar abordagens de grupos específicos da população sobre alimentação 
saudável. Foi lançado na Semana Mundial da alimentação, outubro de 2005, e tem como um dos seus objetivos principais o auxílio ao profissional da saúde na tarefa de informar e educar a população para a adoção de hábitos alimentares saudáveis, tais como o consumo de frutas, verduras e legumes e a diminuição do consumo de sal, de açúcar e de produtos industrializados, para assim evitar o desenvolvimento da obesidade e de outras doenças não transmissíveis.

Durante o debate deste tema nas oficinas, foram observadas expressões de desconhecimento por parte de alguns participantes em relação às publicações referidas, associadas às falas de que até então só conheciam a capa do guia e que em muitas unidades nunca havia sido usado e apresentado à equipe da sua unidade de saúde. Os profissionais foram, portanto, estimulados a recorrer às publicações como um instrumento a ser utilizado no cotidiano de trabalho de vigilância alimentar e nutricional na atenção básica.

Temas específicos como a alimentação e nutrição da gestante demandaram a elaboração de material complementar por parte da equipe de educadoras. Foi percebida a necessidade de publicações sobre a nutrição na gestação, sobre a antropometria no período gestacional entre outras questões do campo da alimentação, tanto nos aspectos biológicos quanto emocionais, que se manifestam durante a gravidez.

As discussões em relação ao material de apoio à vigilância alimentar e nutricional estabelecidas durante o processo educativo levantaram algumas questões relevantes: as publicações disponibilizadas pelo Ministério da Saúde para apoiar a educação nutricional na atenção básica nem sempre chegam ao seu destino final, a unidade de saúde. Ou ainda, chegam e muitas vezes são arquivados antes de serem devidamente utilizados pelas equipes.

As equipes das unidades de saúde têm demandas particulares eminentes de questões socioculturais locais. A elaboração das publicações da CGPAN é idealizada como política global, de caráter geral para a população brasileira. Uma política que ainda não consegue contemplar inúmeras especificidades de cada região, de cada município brasileiro, de cada bairro ou comunidade local.

Nesse sentido, podemos dizer que as propostas apresentadas nos guias do Ministério da Saúde e nas diretrizes da PNAN estão distantes da prática cotidiana na atenção básica à saúde. Essa é uma crítica que alimenta o debate sobre a dificuldade efetiva de tornar a atitude de vigilância nutricional uma realidade nacional, diante das diferenças locais e regionais de grandes extensões geográficas e socioculturais $[22,23,24]$.

No entanto, na fase de avaliação desta experiência, a partir das entrevistas posteriores às oficinas, foram identificados aspectos positivos sobre a utilização das referidas publicações. Os profissionais entrevistados referiram que as publicações foram desarquivadas e assim alcançaram uma certa aplicação prática no cotidiano de algumas equipes.

Outro aspecto revelado na segunda fase da experiência foi em relação à fixação dos cartazes de antropometria publicados pela CGPAN. Durante a visita para realização das entrevistas, verificou-se que os cartazes com técnicas padronizadas para aferição de peso e altura, haviam sido fixados em parede por algumas equipes que antes mantinham o material guardado. Isso indica que pode ter ocorrido o desenvolvimento das habilidades práticas do campo $\mathrm{da}$ antropometria e avanço na atitude e nas atividades de vigilância nutricional.

\section{A metodologia das oficinas e as dificuldades operacionais}

Questões relacionadas ao método aplicado foram identificadas na análise dos registros feitos durante as oficinas. $\mathrm{O}$ envolvimento dos participantes, a linguagem lúdica e o constrangimento diante da proposta vivencial do método aplicado e a conquista de espaços de discussão de questões do cotidiano de trabalho foram algumas das questões emergentes.

A melhora no relacionamento interpessoal foi observada a partir do envolvimento efetivo do grupo nas discussões e nas propostas de atividade que ocorreram nas oficinas. Os participantes reconheceram a importância de trabalhar em grupo, tendo a oportunidade de conhecer e interagir com novas pessoas.

A metodologia participativa das oficinas proporcionou também espaços de discussão e de construção compartilhada de conhecimentos entre os coordenadores das oficinas e os profissionais de saúde. Os participantes compartilharam suas experiências profissionais, as quais foram discutidas e debatidas durante o processo de realização das oficinas. 
A interação entre profissionais de diferentes graus de escolaridade e hierarquia foi reconhecida como um ganho para os grupos. No entanto, alguns grupos mais resistentes apresentavam certa timidez, que pode estar relacionado às distintas categorias profissionais existentes na composição dos grupos. Por exemplo, a participação de coordenadores de unidades de saúde, técnicos, auxiliares de enfermagem e agentes comunitários de saúde.

A superação da inibição para falar dos problemas com outros profissionais durante as oficinas foi relembrada na segunda fase da experiência. Nas entrevistas de avaliação esse tópico foi recordado como um ganho na interação e conhecimento pessoal:

"Gostei por ter sido uma interação entre as pessoas, não havia hierarquia, ninguém era superior e com isso não me constrangia para fazer perguntas" (CGB1).

"Senti à vontade para expor minhas dúvidas, trocar ideias com os colegas da área que estavam participando das oficinas" (EGB1).

Nas oficinas a linguagem lúdica foi utilizada como um recurso no processo de aprendizagem com o intuito de promover de maneira recreativa o estímulo à construção de um novo conhecimento e despertar o interesse pelo tema. $\mathrm{O}$ aprendizado foi facilitado pela utilização desta linguagem com contribuições para a compreensão e aproveitamento dos temas abordados.

Estudos semelhantes mostram que nas atividades lúdicas o educando explora muito mais sua criatividade, melhora sua conduta no processo de ensino-aprendizagem e sua autoestima. Por meio de atividades lúdicas é possível expressar, assimilar e construir a sua realidade. Dessa forma, a ludicidade auxilia na aprendizagem das diversas disciplinas e atividades diárias [25].

A proposta lúdica foi lembrada na segunda fase da experiência como um aspecto positivo do processo de aprendizagem. Segurança para exposição pessoal na construção dos saberes, assim como a vivência de momentos de descontração e alegria foram aspectos resgatados na avaliação.

"As oficinas foram desenvolvidas de maneira bem descontraída, me senti à vontade para estar expondo minhas dívidas..."(EGB1).

"Eu gostei muito, pois parecia uma brincadeira e não aprendizagem. Dessa maneira aprendi muito" (AGB6).
Por outro lado, foi observado durante o desenvolvimento das oficinas o constrangimento dos participantes identificado como um obstáculo importante na vivência do grupo. Frente à proposta participativa, lúdica e vivencial, alguns participantes ficaram inicialmente inibidos e pouco motivados a participar de determinadas atividades.

No planejamento das oficinas a rejeição à metodologia foi levada em consideração. O grupo de educadoras procurou contemplar essa questão por meio da aplicação de estratégias lúdicas e de comunicação intrapessoal, de caráter opcional, o que contemplava o objetivo de quebra da inibição, alcançando, portanto, o envolvimento e participação dos sujeitos 8 .

Os depoimentos obtidos seis meses após a realização das oficinas resgataram a vivência do constrangimento inicial nas oficinas, mas também o processo de desinibição, com ganhos pessoais revelados pelos sujeitos.

"Fiquei um pouco constrangida no comeco das dinâmicas, mas depois fui me acostumando e me soltando" (AGB6).

Como descrito na literatura cada novo método empregado nos processos de ensino provocam impactos distintos [20]. A metodologia participativa e o processo de ensino dinâmico, em que há reflexão, construção coletiva do conhecimento pelo educador e que envolve interação do educador e educando mostra-se como um método que promove a aplicação do conhecimento adquirido demandando envolvimento e exposição pessoal. Enquanto que a metodologia expositiva, com apresentação oral, coloca o educando como um ouvinte e não interage com sua experiência pessoal e sua realidade.

A proposta vivencial demandou da equipe de educadoras o acolhimento das questões de exposição pessoal, como consequência do

\footnotetext{
${ }^{8}$ Um exemplo de estratégia lúdica aplicada com os objetivos de descontração, interação grupal e exercício de observação da linguagem corporal foi proposto em um dos encontros em que os participantes formaram duplas e se posicionaram um em frente ao outro, formando duas rodas, cada par em uma delas. A roda do meio permaneceu fixa e a roda de fora caminhou provocando, com isso, a mudança do par na dupla. Em cada mudança o participante observou o que o novo par mudou em si, o que vestia, se usava brincos, pulseiras, se o cabelo estava preso ou solto, etc. Nessa atividade, o estímulo à comunicação, promoveu segurança aos participantes para se exporem e a oportunidade de enfrentarem a timidez.
} 
envolvimento dos participantes. No entanto, outros problemas de ordem operacional tiveram um peso maior e dificultaram o desenvolvimento das atividades na primeira fase desta experiência.

Embora o projeto tenha sido previamente pactuado com os gestores da saúde dos municípios envolvidos na proposta, as condições operacionais para a realização das oficinas não foram propícias. Relatos sobre essa questão foram revelados nas duas fases da experiência. Em relação à infraestrutura, alguns participantes manifestaram dificuldades de locomoção até o local das oficinas, que impossibilitou a participação deles em um ou mais encontros.

Os espaços físicos adequados para a realização das atividades educativas vivenciais nem sempre estiveram disponíveis. Os horários e os dias estabelecidos para realizar as oficinas nem sempre eram adequados a todos, seja pelo fato da oficina acontecer no último dia útil da semana para alguns grupos ou pelo horário de encerramento impossibilitar a volta ao seu município de origem com o transporte disponibilizado.

$\mathrm{Na}$ fase avaliativa desta experiência essas dificuldades de infraestrutura foram recordadas pelos entrevistados. Dificuldades que disseram respeito principalmente à locomoção, mas, também em relação à falta de comunicação entre os membros da equipe para decidir quem iria participar da oficina.

“... não tínhamos meio de locomoção para chegar até lá. Dependíamos dos carros da prefeitura estarem disponiveis e da autorização para poder utilizá-los” (TGB2).

"Não participamos das oficinas... mas acho que todos nós deveriamos ter participado... Porque é muito importante ter conhecimentos novos que auxiliem em nosso trabalho" (TGB2).

Em estudo realizado por Silva et al. [20] no município de São Carlos foram encontradas questões semelhantes que dificultaram a adesão às oficinas de capacitações com profissionais da rede de saúde do município. A falta de estímulos financeiros, falta de um plano de cargos e salários, a longa duração, dias inadequados, necessidade de custear o transporte, a ocorrência de cursos fora do horário de expediente e a deficiente infraestrutura, que envolveram a falta de organização e as condições do local da capacitação são apresentados pelos autores.

O reconhecimento da contribuição do processo educativo na forma de oficina foi indiscutivelmente o ganho maior desta experiência.
No entanto, a fragilidade do envolvimento dos gestores para evitar a ocorrência das falhas operacionais e de comunicação descritas nesta experiência revela a necessidade de ampliar o processo avaliativo para além das equipes locais.

Devemos considerar ainda, a necessidade de reformular as estratégias de negociação com os gestores do setor público da área da saúde, dos projetos de vigilância alimentar e nutricional na atenção básica, para alcançar melhores condições de continuidade. Estratégias que favoreçam a superação de dificuldades operacionais, mas também minimizar os reflexos da recomposição da administração pública local e regional, ocorridas em função do processo eleitoral e vivenciadas entre a primeira e segunda fase desta experiência.

\section{O campo de conhecimentos da nutrição e a inserção do nutricionista na atenção básica}

A partir da análise das entrevistas realizadas na etapa de avaliação desta experiência foram identificadas duas questões emergentes que permearam os conteúdos dos discursos dos sujeitos: significados atribuídos aos conhecimentos em nutrição na prática cotidiana da atenção primária e o papel do nutricionista nesse contexto.

Os profissionais entrevistados demonstraram motivação e interesse em saber mais sobre nutrição. Essa motivação, no entanto, foi predominantemente vinculada ao autocuidado, o que foi evidenciado pela busca por informações para melhorar a própria alimentação e de seus familiares, maior do que o interesse em aprimorar a prática profissional neste aspecto.

"Sim, influenciou para melhor, pois conbeci a importância de uma alimentação saudável” (EGB6).

"Se eu fizesse tudo correto em relação à alimentação seria mais saudável... tenho que me cuidar mais" (AGB1).

"Gostaria de ter um conhecimento melhor sobre o assunto... e acho que seria bom para mim, pois melhoraria meus hábitos, pois como rapidamente por causa da correria do dia-a-dia, mas tenho o conhecimento que isso não faz bem" (AGB1).

Resultados semelhantes foram identificados no estudo piloto do projeto em que se insere a experiência aqui relatada. O saber em nutrição foi identificado como sendo essencial à vida, fundamental para a saúde, base para o 
desenvolvimento saudável, com significado de grande potência para melhorar a qualidade de vida individual e coletiva [26]. No entanto, a dificuldade de estabelecer mudanças de hábitos, tanto por parte dos próprios profissionais quanto dos pacientes transforma a "boa alimentação" em uma meta difícil de ser atingida no cotidiano.

Em relação à prática do nutricionista, os entrevistados na segunda fase da experiência identificaram que o papel de dar apoio aos membros da equipe na assistência nutricional e alimentar é uma especificidade deste profissional. A atuação do nutricionista na atenção básica, na opinião do grupo ouvido, poderia aprimorar o aconselhamento alimentar dos pacientes.

Os profissionais entrevistados reconheceram a carência de conhecimento técnico e científico em temas vinculados ao campo da nutrição. Cuidados com as doenças crônicas de adultos (hipertensão, diabetes e obesidade), puericultura e desnutrição infantil foram apontados por eles.

“... às vezes não sei orientar corretamente as mães sobre certos alimentos, por não ter o conhecimento técnico sobre o assunto" (TGB2).

"Gostaria de aprender mais sobre o assunto, tanto para mim como em meu trabalho... faço atendimento das crianças, às vezes não sei orientar corretamente" (TGB5).

"Trabalho com crianças procuro estar sempre atenta à nutrição infantil...quero conbecer mais desnutrição e outras doenças para auxiliar no meu trabalho" (AGB6).

"Em saber mais como orientar os pacientes obesos, diabéticos, hipertensos e pacientes em geral que necessitam de uma orientação nutricional” (EGB1).

Segundo Boog [9], profissionais que atuam na rede básica de saúde apresentam dificuldade para atuar com questões do campo da alimentação e nutrição. $\mathrm{O}$ estudo realizado por essa autora identificou que mudanças efetivas de hábitos alimentares necessárias para promover a segurança alimentar demandam esforço conjunto de profissionais que tenham conhecimentos técnicos de epidemiologia, nutrição, dietética e métodos adequados para abordar os problemas nutricionais.

O discurso feito por alguns entrevistados na segunda fase da experiência sugere que com a atuação do nutricionista as mudanças de hábitos alimentares poderiam ocorrer com mais facilidade.
Ao vislumbrar o nutricionista como um membro da equipe local os entrevistados deram sinais de que as orientações dietéticas seriam mais valorizadas pelos pacientes e que estes conseguiriam aderir ao tratamento. Confiança e segurança foram os atributos reconhecidos no nutricionista para a realização dessas demandas identificadas por um conjunto de sujeitos.

Em contrapartida, outros profissionais revelaram que o médico na unidade básica de saúde tem conhecimentos suficientes para assumir todas as demandas e atividades que envolvem o campo da nutrição. Nessa posição o nutricionista deixa de ser indicado para compor a equipe de atenção básica.

\section{CONSIDERAÇÕES FINAIS}

Os temas abordados nas oficinas auxiliaram uma maior compreensão sobre a contribuição da nutrição na vigilância nutricional e possibilitaram melhoria nas orientações nutricionais realizadas pela equipe de atenção básica, as quais ocorrem muitas vezes na ausência do profissional nutricionista. Percebemos no desenvolvimento das oficinas que a antropometria não é realizada de forma padronizada e com as técnicas adequadas, preconizadas no manual do SISVAN. No entanto, após as oficinas, a importância que a atitude de vigilância nas intervenções na realidade da unidade e para as políticas públicas foi percebida pelos participantes desta experiência.

A partir da experiência relatada podemos ressaltar que a metodologia utilizada nas oficinas proporcionou um envolvimento efetivo das equipes de saúde com os profissionais participantes do projeto e com a construção compartilhada de conhecimento, o que foi facilitado pela linguagem lúdica e participativa. Esse método auxiliou na aproximação do conceito teórico com a realidade vivida, porém, os profissionais demonstraram constrangimento ao se depararem com a proposta participativa, lúdica e vivencial, superado após o desenvolvimento de dinâmicas de grupo, conforme os depoimentos registrados.

Aspectos operacionais no desenvolvimento das oficinas tais como a falta de espaço físico adequado; infraestrutura para locomoção das equipes e carga horária dos profissionais para se dedicarem ao processo educativo foram considerados obstáculos importantes nesta experiência. Problemas de comunicação entre os articuladores do projeto, as equipes e coordenadores das oficinas foram igualmente observados no seu desenvolvimento. 
Concluímos que há necessidade de maior investimento e apoio por parte dos gestores e parceiros para a consolidação da promoção da atitude de vigilância alimentar e nutricional como prática cotidiana e também como diretriz política da atenção básica no contexto do Sistema Único de Saúde no Brasil. Uma atitude que deve ser desenvolvida por meio de trabalho coletivo da equipe e fomentada por políticas públicas setoriais e locais de Segurança Alimentar e Nutricional.

Há de se considerar que a atitude de vigilância nutricional é uma questão central na atenção básica que demanda capacitação da equipe com apoio de nutricionistas. A importância deste profissional na atenção básica foi reconhecida pelos profissionais que participaram desta experiência, porém nem sempre esta questão é consensual entre os membros da equipe básica do modelo vigente de atenção básica em saúde.

\section{AGRADECIMENTOS}

Os autores agradecem o Apoio Financeiro recebido da Fundação de Amparo à Pesquisa do Estado de São Paulo - FAPESP e do Ministério da Saúde e Ministério da Ciência e Tecnologia - Convênio FAPESP/CNPq/SUS.

\section{REFERÊNCIAS}

[1] Popkin BM. The nutrition transition and obesity in the developing world. J Nutr. 2001;131(3):871-873.

[2] Engstrom EM, Anjos LA. Déficit estatural nas crianças brasileiras: relação das condições sócio-ambientais e estado nutricional materno. Cad Saude Publica. 1999;15(3):559-567.

[3] Sigulem DM, Devincenzi MU, Lessa AC. Diagnóstico do estado nutricional da criança e do adolescente. J Pediatr. 2000;76(8):275-284.

[4] The World Bank. Repositioning Nutrition as Central to Development: A strategy for largescale Action [internet]. Washington, 2006 [cited 2008 may 10]. Available from: http://www.worldbank.org

[5] Ferreira HS. Desnutrição: magnitude, significado social e possibilidades de prevenção. Maceió: EDUFAL; 2000.

[6] Brasil. Ministério da Saúde. Secretaria de Atenção à Saúde. Departamento de Atenção Básica. Política nacional de alimentação e nutrição / Ministério da Saúde, Secretaria de Atenção à Saúde, Departamento de Atenção Básica - 2a ed. rev. Brasília: Ministério da Saúde; 2008. 48 p. (Série B. Textos Básicos de Saúde).
[7] Brasil. Ministério da Saúde. Vigilância alimentar e nutricional - Sisvan: orientações básicas para a coleta, processamento, análise de dados e informação em serviços de saúde / [Andhressa Araújo Fagundes et al.]. Brasília: Ministério da Saúde; 2004. 120 p. (Série A. Normas e Manuais Técnicos).

[8] Campos SH, Boog MCF. Cuidado nutricional na visão de enfermeiras docentes. Rev Nutr. 2006;19(2):145-155.

[9] Boog MCF. Dificuldades encontradas por médicos e enfermeiros na abordagem de problemas alimentares. Rev Nutr. 1999;12(3):261-272.

[10] Brasil. Ministério da Saúde. Secretaria de Atenção à Saúde. Coordenação-Geral da Política de Alimentação e Nutrição. Trabalhos apoiados pela Coordenação-Geral da Política de Alimentação e Nutrição. Brasília: Ministério da Saúde; 2006. 142 p. (Série C. Projetos, Programas e Relatórios).

[11] Instituto Brasileiro de Geografia e Estatística. Perfil demográfico (2008). Brasília: Fundação IBGE; 2008 [acesso em 21 set 2009]. Disponível em http://www.ibge.gov.br

[12] Afonso MLM, Coutinho ARA. Metodologias de trabalho com grupos e sua utilização na área da saúde. In: Afonso MLM (org.). Oficinas em dinâmica de grupo na área da saúde. Itatiba, SP: Casa do Psicólogo; 2006. P. 5983.

[13] Brasil. Ministério da Saúde. Secretaria de Gestão do Trabalho e da Educação na Saúde. Departamento de Gestão da Educação em Saúde. Política Nacional de Educação Permanente em Saúde . Brasília: Ministério da Saúde; 2009. 64 p. (Série B. Textos Básicos de Saúde).

[14] Brasil. Ministério da Saúde. Secretaria de Atenção à Saúde. Departamento de Atenção Básica. Alimentação e nutrição para as famílias do Programa Bolsa Família: manual para os agentes comunitários de saúde. Brasília: Ministério da Saúde; 2007. 52 p. (Série A. Normas e Manuais Técnicos).

[15] Brasil. Ministério da Saúde. Secretaria de Atenção à Saúde. Coordenação-Geral da Política de Alimentação e Nutrição. Guia alimentar para a população brasileira: promovendo a alimentação saudável. Brasília: Ministério da Saúde; 2005. 236p. (Série A. Normas e Manuais Técnicos).

[16] Rusness B. Striving: for empowerment thought nutrition education. J Am Diet Assoc. 1993;93(1):78-79. 
[17] Verdi M, Caponi S. Reflexões sobre a Promoção da Saúde numa perspectiva bioética. Texto Contexto Enferm. 2005;14(1):82-88.

[18] Vieira CM, Codeiro SN, Magdaleno Júnior R, Turato ER. Significados da dieta e mudanças de hábitos para portadores de doenças metabólicas crônicas: uma revisão. Ciência Saúde Colet. 2009 [acesso em 25 out 2010]. Disponível em: http://www.cienciaesaudecoletiva.com.br/artigos/artigo int.php?id artigo $=4247$

[19] Brasil. Ministério do Desenvolvimento Social e Combate à Fome. Como feijão com arroz seu dia-a-dia muito bem acompanhado. Brasília: Ministério do Desenvolvimento Social e Combate à Fome; 2008 [acesso em 21 set 2009]. Disponível em: http://www.mds.gov.br/brasilquedagosto/materiais-dedivulgacao

[20] Silva JAM, Ogata MN, Machado MLT, Capacitação dos trabalhadores de saúde na atenção básica: impactos e perspectivas. REE. 2007;9(2):389-401.

[21] Fernandez PMF, Voci SM, Kamata LH, Najas MS, Souza ALM. Programa Saúde da Família e as ações em nutrição em um distrito de saúde do município de São Paulo. Ciência Saúde Colet. 2005;10(3):749-755.

[22] Silva DO, Recine EGI, Queiroz EFO. Concepções de profissionais de saúde da atenção básica sobre a alimentação saudável no Distrito Federal. Cad Saude Publica. 2002;18(5):1367-1377.

[23] Batista FM, Rissin A. Vigilância alimentar e nutricional: antecedentes, objetivos e modalidades. A VAN no Brasil. Cad Saude Publica. 1996;9(1):99-105.

[24] Arruda BKG. Sisvan: breve viagem ao passado. In. Apresentado na Mesa Redonda "Sisvan - Histórico, Avaliação e Perspectivas, do VI Encontro Nacional dos coordenadores Estaduais e Centros Colaboradores em Alimentação e Nutrição". Brasília; 2006.

[25] Neves LOR. O lúdico nas interfaces das relações educativas Rondônia: Centro de Referência Educacional [monografia da internet]. 2007 [acesso em 14 jul 2009]. Disponível em: http://www.centrorefeducacional.com.br/ludicoint.htm

[26] Oliveira MRM, Vieira CM. Avaliação da implementação das ações de vigilância alimentar no âmbito da atenção básica do SUS na região das bacias Piracicaba-Capivari [Relatório]. Piracicaba: Universidade Metodista de Piracicaba; 2007. 